\title{
ENIUS. European Network of multidisciplinary research to Improve the Urinary Stents. COSTS Actions. CA16217. European Cooperation in Science \& Technology
}

Selected Abstracts from the "Advances in Urinary Stents. Biomaterials, Coatings and Drug Eluting Stents". Training School Meeting. Name of Meeting Organizer. European Network of multidisciplinary research to Improve the Urinary Stents (ENIUS). COSTS Actions. CA16217. European Cooperation in Science \& Technology. University of Lublin (Poland), 26th-27th September, 2019.

Selected Abstracts from the "Advances in Urinary Stents technology". Workshop. Name of Meeting Organizer. European Network of multidisciplinary research to Improve the Urinary Stents (ENIUS). COSTS Actions. CA16217. European Cooperation in Science \& Technology.

Serbian Academy of sciences and Arts, Belgrade (Serbia), 7th March, 2020.

Sponsorship: Publication of this supplement was funded by ENIUS COST Action. All content was reviewed and approved by the Society Committee, which held full responsibility for the abstract selections. Dr. Federico Soria (Spain).

ENIUS network (2017-2021) bring together European translational scientists, urologists, bioengineers, chemists, to foster the dialogue and exchange expertise and to contribute to enhancing the urinary stents and reduce the morbidity of stented patients. Currently, ENIUS is composed of 262 researchers from 36 different countries. It is the largest research network in urinary stents in the world. ( http://www.enius.org).

COST provides networking opportunities for researchers and innovators in order to strengthen Europe's capacity to address scientific, technological and societal challenges.

The three strategic priorities:

- Promoting and spreading excellence.

- Fostering interdisciplinary research for breakthrough science.

- Empowering and retaining young researchers and innovators.
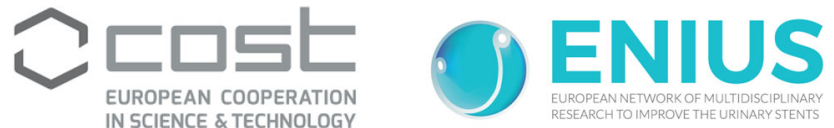

1. Advances in Urinary Stents. Biomaterials, Coatings and Drug Eluting Stents (DES). Training School. 26th-27th September, 2019. Lublin (Poland).
2. Advances in Urinary Stents technology. Workshop. 7th March, 2020. Belgrade (Serbia).

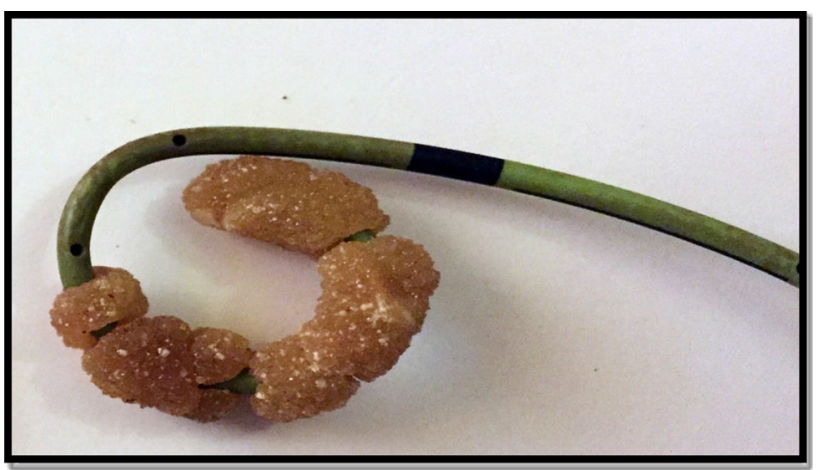

1. Preliminary assessment of the MMC BraidStent ${ }^{\circledR}$ : a mitomycin C-eluting biodegradable ureteral stent

Julia E. de la Cruz ${ }^{1 *}$, Maria Soto ${ }^{1}$, Alberto Budía ${ }^{2}$, Juan Pablo Caballero ${ }^{3}$, Francisco Sánchez-Margallo ${ }^{1}$, Federico Soria ${ }^{1}$

${ }^{1}$ Jesús Usón Minimally Invasive Surgery Centre, JUMISC, Caceres, Spain; ${ }^{2}$ Urology Department, La Fe University Hospital, Valencia, Spain; ${ }^{3}$ Urology Department, General University Hospital, Alicante, Spain

The objective of this line of research is the development of a biodegradable drug-eluting ureteral stent, for the topical adjuvant treatment of upper urinary tract urothelial carcinoma: MMC BraidStent ${ }^{\circledR}$. BraidStent ${ }^{\circledR}$ is a biodegradable intra-ureteral stent, whose safety and efficacy in vitro and in vivo has been proved by our research group in previous studies. The efforts of our research nowadays are focused on the development of the polymer that is able to coat the stent and to carry and release Mitomycin C (MMC), at appropriate doses and timing. We have tested in vitro a polymeric matrix of natural origin. The first evaluation included the study of the coating dyed with Sulforhodamine B ( $\mathrm{SrB})$, to assess the feasibility of attachment to the biodegradable materials of the BraidStent ${ }^{\circledR}$. Besides, three different coating techniques for controlled drug 
delivery were analysed assessing the release of methylthioninium chloride in PBS: "dip coating", "nano-coating" and "dip coating mix". The results of this first study showed that the polymeric matrix is able to coat the stent materials and that of the three coating techniques, "dip coating mix" showed a sustained release over $72 \mathrm{~h}$ with the highest mean release concentration.

Subsequently, the release of MMC is evaluated via incubation of coated BraidStent ${ }^{\circledR}$ samples in artificial urine (AU), subjected to orbital agitation with periodic urine exchanges and follow-ups for 30 days. Release kinetics are analysed via HPLC until the 10th-day follow-up. The results of these tests show that the release of MMC occurs during the first $48 \mathrm{~h}$ and that there may be a delay in degradation due to the coating. Nevertheless, more in vitro studies in AU will be performed for the assessment of both the coating and the MMC carrying and kinetics before the consequent evaluation in vivo in the porcine model.

\section{Mathematical modelling of the swelling of biodegradable ureteral stents in vitro}

\section{Nikita Greenidge*}

University of Oxford, Oxford, UK

The biodegradable ureteral stents developed by Hydrustent have the unique ability of degrading homogeneously during deployment, eliminating the need for expensive removal procedures. Mathematical modelling may play a vital role in the acceleration of this product's development as it allows for a reduction in preclinical trials involved. Modelling these biodegradable stents would give us more insight into the mechanism of degradation, allowing us to potentially predict the time taken for degradation and the degree of swelling that occurs.

In this work, the stent being modelled is made up of a gelatinalginate Interpenetrating Polymer Network (IPN) hydrogel. While models have been developed for the swelling of IPN hydrogels, none has looked at this formulation. This model was developed at the University of Oxford (UK) with validation studies being conducted at the 3B's Research Group (Portugal). The model developed is a simplified one-dimensional model in which an attempt was made to capture the major mechanisms involved in the swelling and degradation of the hydrogel. The model assumes that the stent has been placed in artificial urine in a static environment. The preliminary model has shown that it can output the change in dimension of the stent's cross-section over time.

\section{Imaging of urethral stricture disease-a three- dimensional computerized model based on the magnetic resonance urethrography: a novel technique to assess spongiofibrosis}

\section{Mikolaj Frankiewicz* \\ Department of Urology, Medical University of Gdansk, Poland}

The concept of stent application in urological practice is constantly being evaluated to protect patients from symptoms of urethral or ureteral obstruction. After great enthusiasm at the beginning, high complication rates have decreased the initial optimism. Fibrotic stenosed urethras with a stent imbedded in the midst of them with no retrievable urethral wall has been the most serious, yet frequent complication reported within patients after urethral stent implantation. In our research, we are looking for an optimal tool that may be used preoperatively to assess the extent of spongiofibrosis and peri-urethral pathological tissue in order to chose the best treatment for each patient individually. Imaging modalities with three-dimensional (3D) capabilities such as magnetic resonance urethrography (MRU) may provide more comprehensive information regarding urethral diseases through a more detailed illustration of peri-urethral soft tissue structures. Based on prior experience, we also performed MRU in a patient with extensive fibrosis 20 years after implantation of the UroLume endourethral prosthesis (American Medical Systems, Minnetonka, Minnesota) and we have thoroughly analysed the changes responsible for the restenosis found in the urethra.

\section{Physiologically representative urethral models for the evaluation of intermittent urinary catheter lubricity and urethral micro-trauma}

\section{Owen Humphreys*}

School of Mechanical and Materials Engineering, University College Dublin, Dublin, Ireland

Intermittent urinary catheter users typically catheterise 4 times per day. This repeated catheterisation can cause urethral irritation and pain for the users. An anatomically accurate silicone urethral model was created to measure the insertion and extraction friction forces of four commercially available intermittent catheter brands. An ex vivo porcine penis urethral model was also created to investigate the effect of catheters sticking and drying in the urethra. An animatronic hand was designed to grip the catheter and catheterise the urethral models while measuring the friction forces with a load cell. The silicone urethral model showed that it required more force to insert a catheter than to extract it, supporting clinical anecdotes. The effect of catheter eyelets was investigated by catheterising the silicone urethral model with the eyelets in two different orientations. The eyelets positioned perpendicular to the sagittal plane (i.e. horizontally) resulted in lower friction than when positioned parallel to the sagittal plane (i.e. vertically), advising users of this technique may result in less urethral irritation and pain. The porcine urethral model showed all catheter brands tested experienced some degree of sticking in the urethra, however one brand adhered very strongly. Another brand showed evidence of drying in the urethra, resulting in a higher extraction resistance after $10 \mathrm{~min}$ in the urethra compared to $2 \mathrm{~min}$ in the urethra. These benchtop urethral models can be used to aid in the development of new and improved catheter designs, and provide a method for evaluating the effect of catheter eyelets on friction and the effect of the catheter coatings on sticking in the urethra.

\section{The influence of carbon nanotubes/ polydimethylsiloxane composites on bacterial adhesion and biofilm formation}

Rita Teixeira-Santos ${ }^{1 *}$, Marisa Gomes ${ }^{1,2}$, Márcia R. Vagos ${ }^{1}$, Olívia S. G. P. Soares ${ }^{2}$, Manuel F. R. Pereira ${ }^{2}$, Filipe J. Mergulhão ${ }^{1}$

${ }^{1}$ LEPABE-Laboratory for Process Engineering, Environment, Biotechnology and Energy, Faculty of Engineering, University of Porto, Porto, Portugal; ${ }^{2}$ Laboratory of Separation and Reaction Engineering-Laboratory of Catalysis and Materials (LSRE-LCM), Department of Chemical Engineering, Faculty of Engineering, University of Porto, Porto, Portugal

Polydimethylsiloxane (PDMS) is one of the most widely used silicones for the fabrication of medical devices, including the ones from the urinary tract. Urinary stents are commonly used in urology 
practice, improving the patient's quality of life. However, these devices are highly prone to bacterial colonization and biofilm formation, causing urinary tract infections in $31 \%$ of the patients after their insertion. The incorporation of carbon nanotubes (CNTs) in PDMS composites has proved to play an important role in the modulation of bacterial adhesion. The aim of this study was to produce pristine-MWCNT/PDMS composite films and evaluate their effect on bacterial adhesion and biofilm formation. Composites were produced by incorporation of pristine CNT in a PDMS matrix and characterized using scanning electron microscopy. E. coli adhesion and biofilm formation were evaluated using a parallel plate flow chamber under hydrodynamic conditions that mimic the urinary flow in critical areas of urinary stents, which are prone to encrustation. For the carbon nanotubes composites, bacterial adhesion rate was lower than for the control (PDMS). Additionally, after $30 \mathrm{~min}$, composites containing a small concentration of pristine carbon nanotubes $(0.1 \%)$ reduced E. coli adhesion by $20 \%$ compared to the control ( $<<0.05)$. Results suggest that bacteria adhesion can be inhibited by the incorporation of CNT in a PDMS matrix. These findings may be promising for the development of new coating surfaces to prevent and control the bacterial adhesion and biofilm formation in urinary stents.

\section{Necessity of improving urinary stents used during pregnancy}

\section{Martina Ambardjieva*, Maja Sofronievska Glavinov \\ University Surgical Clinic "St. Naum Ohridski”, Department of urology, Skopje, Republic of North Macedonia}

The purpose of this study is to determine the effects of intubation, the impact of complications and to estimate the necessity of improving urinary stents used during pregnancy. Hydronephrosis during pregnancy most commonly develops as a result of compression of the ureters as well as the effect of progesterone on the smooth muscle relaxation. Clinical significance of hydronephrosis lies in the association between ureteral obstruction and the high frequency of ascending urinary tract infection during pregnancy. Most of the cases of symptomatic hydronephrosis in pregnancy resolve with conservative measures with a rare need of stent insertion. When needed, ureteric stents are inserted with local anaesthesia under transabdominal US guidance. Pain and discomfort are common experiences during the procedure. The most common complication of ureteric stents is encrustation which occurs in this population likely secondary to the changes in urinary chemistry during pregnancy, such as hypercalciuria and hyperuricosuria. Ureteric stents placed in pregnant women are generally exchanged every 4-6 weeks in order to avoid encrustation and potential obstruction, necessitating the repeated cost and morbidity of repeated procedures. Infection and migration are other complications of internal stent replacement. In addition, this repetitive procedure can induce rupture of the intrauterine membranes in some cases, or can induce preterm delivery due to invasive and uncomfortable procedure especially in the third trimester of pregnancy. Sometimes for removal of the ureteric stent performing ESWL is inevitable because severe incrustation prevents stent removal after delivery, especially for patients without periodical replacement of the stents. Multiple procedures, morbidities, antibiotics, dietary calcium restriction and pain associated with ureteral stents replacement can all have a negative impact on a pregnancy. For these reasons, biodegradable stents should be preferred and are ideal solution in the properly selected pregnant patient, particularly as this device technology continues to improve.

\section{Bacterial adhesion to catheter surfaces}

\section{Klemen Bohinc*}

Faculty of Health Sciences, University of Ljubljana, Ljubljana, Slovenia

Interactions between bacteria and different types of surfaces play an important role in different disciplines of medicine. Urinary tract infection associated with application of catheters is one of the most common infections in hospitals and health care facilities that are also a great financial burden to the health care. The catheter surface properties and liquid flow properties dictate the conditions for bacterial adhesion. The aim of our studies is to investigate the influence of catheter surface characteristics on the bacterial adhesion rate. We consider different types of catheter surfaces with different surface modifications. The roughness, hydrophobicity, morphology and texture of the material surfaces were measured. The adhered E. coli bacteria on catheter surfaces were stained and spectrophotometrically detected. The results were also verified by scanning electron microscopy. At the end of my presentation the outlook for future studies including polyelectrolyte coatings will be made.

\section{Thin and biocompatible nano films as protection for in vivo pressure sensors}

\section{Ingelin Claussen*}

Uro Bionics, Norway

Micro Electro Mechanical Systems (MEMS) technology in combination with advanced nanotechnology can revolutionize the field of medical diagnosis and treatment. Applying such technology in the development of in vivo sensor systems enables direct and continuous organ monitoring providing high-quality data.

In the human body, pressure is an essential parameter in almost all organs. Reliable in vivo pressure sensors are therefore strongly desired. For long-term monitoring in the human body, however, biocompatibility represents a major obstacle for the success. Not only must the impact of the device on the body be handled (toxicity, mechanical impact, inflammation, wear and degradation), but also the body's reaction to the device (rejection, encapsulation, biofouling etc.). For sensors used in vivo, corrosion caused by aggressive body fluids and accumulation of biological matters (urea, proteins, cells etc.) may alter the sensor characteristics and thereby the sensor stability. Some of the immunological responses can be overcome by smart sensor design. Others can be dealt with using a protective package. Traditional MEMS packages are relatively massive housings of metal or solid polymers and will in many cases spoil the opportunities for true miniaturization. A thin biocompatible coating might provide adequate protection against biological attack and at the same time maintain the small-size advantage. Still, the coating must also be compatible with the sensor's functionality.

I have for several years examined biocompatible coatings of nanoscale thickness as a substitute for the traditional MEMS packages. This work has been crucial for the development of an in vivo pressure measurement system for monitoring of urinary bladder pressure. I will present some of the results and further present requirements for the ultimate coating to provide for a permanently implantable sensor system for monitoring of urinary bladder pressure. 


\section{Fighting bacterial adhesion with antibiotic-free solutions}

\section{Fabiola Costa*}

i3S-Instituto de Investigação e Inovação em Saúde, Porto Universidade, Portugal

Ureteral stents are frequently used medical devices, particularly prone to infection and related complications. Current strategies to prevent this problem based on device coatings with antimicrobial compounds (antibiotics or antiseptics) have proven to be insufficient, often toxic, and even promoting bacterial resistance. Herein, we report antibioticfree alternative coatings to mitigate this problem. CyanoCoating is an infection preventive coating based on natural polymer released by the marine cyanobacterium. At the proof-of-concept stage we have proved efficacy against a wide spectrum of microorganisms, nontoxicity and scalability to different substrate materials (polyurethane and silicone). CyanoCoating's mode-of-action is based on a nonpharmacologic effect that impairs microbial adhesion to the surface hindering the first steps of biofilm-formation. A second approach is based on the application of proprietary antimicrobial peptides (ChainD) on to coatings, as these new classes of alternatives to antibiotics enables biofilm disruption, without aggravating the rising problem of microbial resistance.

\section{Method of post-manufacture impregnation to create a drug-eluting stent}

\author{
Beatriz Domingues ${ }^{1,2 *}$, Ivo Aroso ${ }^{1,2}$, Estevão Lima ${ }^{4}$, \\ Alexandre Barros ${ }^{1,2}$, Rui L. Reis ${ }^{1,2,3}$ \\ 13B's Research Group, I3Bs-Research Institute on Biomaterials, \\ Biodegradables and Biomimetics, University of Minho, Guimarães, \\ Portugal; ${ }^{2}$ ICVS/3B's-PT Government Associate Laboratory, Braga/ \\ Guimarães, Portugal; ${ }^{3}$ The Discoveries Centre for Regenerative \\ and Precision Medicine, Headquarters at University of Minho, \\ Guimarães, Portugal; ${ }^{4}$ Life and Health Sciences Research Institute \\ (ICVS), School of Health Sciences, University of Minho, Braga, \\ Portugal
}

The improvement of medical devices through material design and development is a constant need. Within the urological field, medical devices are associated with several complications including symptomatic bacterial infections and catheter blockage due to biofilms and encrustation, which is formed by deposition of salts and minerals. Over the years, ureteral device technology has evolved, in order to improve the safety and the patient's comfort. Their design is to obtain improvement in anti-bacterial surfaces, using, for instance, hydrogelcoated stents, with resistance, to some extent, to bacterial infection and encrustation. Oral agents, a commonly used strategy for stent discomfort, are associated with possible side-effects and minimal efficacy. To overcome this, stent surfaces that allow the local delivery of drugs have been developed and represent a valuable alternative, promoting an efficient treatment and preventing any side-effects. This type of technology has been widely used in the treatment of cardiovascular diseases, and its beneficial results substantiate the importance of using it also for treatments in the urinary system. In this project, we developed an improved impregnation method able to create a drug-delivery stent with enhanced features. The method of post-manufacture impregnation did not damage the commercial biomaterial used and, from the mechanical tests, it was concluded that, among other things, the tensile strength is affected by less than $10 \%$. After this impregnation process, stents showed controlled drug delivery while maintaining the pharmacological properties over time.
This drug-eluting technology seems to be a promising option to help reducing the most frequent complications and to improve the comfort of patients.

\section{Marine biofilm formed by coccoid cyanobacteria: development and control}

\section{Sara Isabel Faria}

LEPABE-Department of Chemical Engineering, Faculty of Engineering, University of Porto, Portugal

Marine biofouling is an ongoing concern in which cyanobacteria play a pivotal role. Although it has been recognized that the hydrodynamic and the surface properties can seriously affect adhesion and biofilm development, their impact on cyanobacterial biofilms has rarely been evaluated. Therefore, the first goal of this work was to understand the effect of these two parameters - hydrodynamic conditions and surface properties - on cell adhesion and biofilm formation by using marine cyanobacteria from the Portuguese coast in controlled hydrodynamic conditions which mimic the marine environment, and two representative surface materials, namely glass and gel-coated glass.

Despite the negative impact of cyanobacteria in marine biofouling, it has been shown that some strains produce biological compounds with antifouling properties. Thus, their potential against emerging biofilms is an attractive research area. In the second part of the study, cyanobacterial extracts were tested for their ability to reduce already formed marine biofilms. At a later stage, these extracts will be tested for application in biomedical surfaces, such as urinary catheters.

\section{Polyurethane-based hydrogels as promising drug- loaded coatings for biomedical devices}

\section{Rossella Laurano*, Monica Boffito, Valeria Chiono, Gianluca Ciardelli}

Politecnico di Torino, Department of Mechanical and Aerospace Engineering, Turin, Italy

Recently, research into new functional biomaterials integrated with biomedical devices has become a challenging issue aimed at improving the immune system response. In this regard, polyurethane (PU) biomaterials are gaining increasing interest due to the high versatility of their chemistry, which opens up the possibility to modulate their physico-chemical and mechanical properties by simply changing their building blocks. In this work, an amphiphilic PU was successfully synthesised [Boffito et al. 2016, https://doi.org/10.1002/pi.5080] starting from Poloxamer ${ }^{\circledR}$ P407 (poly(ethylene oxide)-poly(propylene oxide)-poly(ethylene oxide) PEO-PPO-PEO triblock copolymer, $70 \%$ PEO, $\mathrm{M}_{n} 12,600 \mathrm{Da}$ ) as 1,6hexane diisocyanate or Hexamethylene diisocyanate and N-Boc diethanolamine as a chain extender for the design of thermosensitive hydrogels for drug delivery applications. The characteristic amphiphilic PEU molecular organisation into micelles in response to a temperature increase allowed the encapsulation of drugs with different wettability (i.e., hydrophobic drugs inside the micelle core, hydrophilic drugs within the interstitial space between micelles). Drug encapsulation did not induce changes in gelation conditions (sol-gel transition at $37{ }^{\circ} \mathrm{C}$ within $5 \mathrm{~min}$ ), but different payload release kinetics were observed because of their different loading site. Furthermore, after Boc protecting group cleavage, the high number of -NH groups exposed along the polymer chains was exploited to graft photosensitive molecules (i.e., thiols and acrylate groups). In this way 
it was possible to further increase hydrogel residence time in aqueous environment through crosslinking upon UV or Vis light irradiation. Moreover, hydrogel cytocompatibility was also evaluated according to ISO10993.

Hence, due to their high versatility and easy process-ability, PUbased hydrogels, either physically or chemically cross-linked, can be further exploited as promising drug-containing coatings for biomedical devices, such as urinary of cardiovascular stents and prosthesis, providing a localized and controlled release of active principles for the treatment of infections and inflammation.

\section{Reference}

Boffito M, Gioffredi E, Chiono V, Calzone S, Ranzato E, Martinotti S, Ciardelli G (2016) Novel polyurethane-based thermosensitive hydrogels as drug release and tissue engineering platforms: design and in vitro characterization. Polym Int 65:756-769. https://doi.org/10.1002/pi.5080

\section{Curcumin impregnation by supercritical fluid technology in silicon-based ureteral stents}

\author{
Margarida Pacheco $^{1 *}$, Joana M. Silva ${ }^{1,2}$, Ivo Aroso ${ }^{1,2}$, \\ Eduardo Silva $^{1,2}$, Estevão Lima ${ }^{2,3}$, Alexandre Barros ${ }^{1,2}$, \\ Rui L. Reis ${ }^{1,2}$ \\ 13B's Research Group-Biomaterials, Biodegradables \\ and Biomimetics, University of Minho, Guimarães, Portugal; ${ }^{2}$ ICVS/ \\ 3B's-PT Government Associate Laboratory, Braga/Guimarães, \\ Portugal; ${ }^{3}$ Life and Health Sciences Research Institute (ICVS), \\ School of Health Sciences, University of Minho, Braga, Portugal
}

Nowadays, ureteral stenting is an everyday procedure in urological practice. However, despite its proved indispensable role, researchers on this area face some major challenges, namely stent encrustation and infection, both promoted by bacterial colonization. Even though many strategies have been implemented with some success, such as the integration of antibiotics, the maintenance of an efficient release and the preservation of the antibiotic efficiency during the whole treatment has been a major challenge. Herein, we propose a strategy based on the local release of an antibacterial compound, curcumin, to overcome the bacterial colonization problem on ureteral stents. Curcumin is known to be effective against multidrug resistant bacteria and by possessing anti-biofilm activity. Indeed, its inhibitory effects against a broad spectrum of bacteria have already been reported. In this work, we successfully impregnated curcumin into silicon ureteral stents (Coloplast) using supercritical fluid $\mathrm{CO}_{2}\left(\mathrm{scCO}_{2}\right)$ (100 bar, $50{ }^{\circ} \mathrm{C}, 12 \mathrm{~h}$ ) with no influence in the stent morphology. The amount of the antibacterial compound loaded was determined by UV-Vis $(428 \mathrm{~nm})$, by immersing the stent in different solvents. The antibacterial activity test revealed that for $E$. coli, this method does not show significant efficiency, while for $S$. aureus, some growth inhibition was noted. To evaluate the cytotoxic effect, L929 cells were used as a model.

The overall results showed that curcumin was successfully impregnated into the silicon stents and some antibacterial effect was achieved. Even though the technique to measure the curcumin amount impregnated should be optimized, and the antibacterial and cytotoxic activity must be improved, taking into account the curcumin amount that exerts both effects, this study demonstrates the potential of this approach for the improvement of urinary stents. In future, it is intended to test the anti-tumour activity of the curcumin-loaded stents.

\section{Effectiveness of novel anti-biofilm strategies on urinary stents: the importance of hydrodynamic conditions}

\section{Maria João Romeu*, Filipe Mergulhão}

Faculdade de Engenharia, Laboratorio do Processos, Ambiente,Biotecnologia e Energia, Universidade do Porto, Portugal

Urinary tract infections represent a major health concern worldwide and the development of biofilms in urinary stents and catheters remains an ongoing issue. Amongst the parameters that affect biofilm development, flow velocity and shear rate are some of the most important. There is evidence that shear forces affect not only biofilm structure, but also composition, mass transfer, exopolysaccharide production and energy metabolism and can also induce genetic or molecular changes in biofilms. Therefore, it is important to perform biofilm formation studies in controlled hydrodynamic conditions in order to mimic real hydrodynamics on medical devices. Although several platforms are available to perform biofilm studies, the use of microtiter plates as high-throughput platforms to mimic hydrodynamics on medical devices is a useful preliminary approach to test different coatings on new surfaces for biomedical application. A detailed hydrodynamic analysis of these biofilm reactors is very useful and Computational Fluid Dynamics is a powerful tool used to model flow situations to assess if the hydrodynamic conditions in vitro are similar to those prevailing in these biomedical devices. The effect of marine compounds on the reduction of $E$. coli biofilms under controlled hydrodynamic conditions was evaluated. This presentation highlights the importance of hydrodynamics on biofilm development and control, as a factor which must be taken into account for validation studies of new compounds and surfaces depending on their application.

\section{Urinary stent-associated symptoms, quality of life and metallic stent failure}

\section{Moisés Rodríguez-Socarras*}

Instituto de Cirugía Urológica Avanzada (ICUA), Madrid, Spain

Aim: To analyze urinary stent-associated symptoms, Quality of Life and Metallic Stents failure.

Method: Comprehensive analysis of the literature on ureteral stents, side effects and Quality of Life (QoL).

Results: Temporary drainage of the upper urinary tract using ureteral stents is a urological procedure to assure renal function and to treat pain and major infections caused by ureteral obstruction. Wrong placement and migration during or after the procedure can occur. Side-effects and stent-associated symptoms are common, including flank pain, haematuria, pain of voiding, incrustation, infections, biofilm formation, lower urinary tract symptoms (LUTS) and impairment of quality of life. However, the pathophysiology of stent-associated morbidity is poorly understood; its prevention and treatment are also limited.

Traditionally, DJ stents should usually be removed or changed within a short-medium time period (around 3-4 months at maximum). Reasons for long-term ureteral stenting include: delay until definitive surgical treatment, ureteral strictures or patients who are unfit for major surgery (e.g. oncology patients) and stents lost in patients for months or even years. Metallic ureteral stents (MUS) can be used for long-ureteral stenting, but the procedure is technically more demanding and feasibility is limited in the case of multiple and long strictures. Furthermore, metallic stent failure may occur cause failure 
in primary patency, stent occlusion, ureteral re-occlusion, stent dysfunction, migration, encrustation, tissue or tumour ingrowth and edgestenosis by urothelial hyperplasia.

Conclusion: Efforts should be done to develop new stents in order to avoid side-effects and preserve patients' quality of life.

\section{Urinary infections associated with lower urinary tract catheters-from evidence to alternatives}

\section{Maja Sofronievska Glavinov* \\ University surgical Clinic “St. Naum Ohridski”, Skopje, Republic of North Macedonia}

Lower urinary tract catheters most commonly cause urinary tract infection that occurs while a patient has an indwelling urinary catheter or within $48 \mathrm{~h}$ of its removal. These infections represents the largest institutional reservoir of nosocomial antibiotic-resistant pathogens such as Escherichia coli multidrug-resistant Enterobacteriaceae (Klebsiella, Enterobacter, Proteus, and Citrobacter), Pseudomonas aeruginosa, enterococci and staphylococci and Candida spp. Technological innovations have been proposed and evaluated during the past 25 years but have not proven conclusively beneficial. Among these innovations are: the use of anti-infective lubricants when inserting the catheter, soaking the catheter in an anti-infective antimicrobial-drug solution before insertion, regular metal cleansing or periodically applying anti-infective creams or ointments to metals, continuously irrigating the catheterized bladder with an anti-infective solution through a triple-lumen catheter, or periodically instilling an anti-infective solution into the collection bag. Bladder irrigation with antimicrobial-drug solutions has not only shown no benefit for prevention but has been associated with a strikingly increased proportion of UTIs caused by microorganisms resistant to the drugs in the irrigating solution. Present evidence in the literature regarding catheter materials indicates that there is no significant difference between antimicrobial catheter materials versus standard materials for shortterm catheterization. There is no clear benefit among standard materials on infection rates including: latex, hydrogel-coated latex, silicone-coated latex, or all-silicone catheters. The evidence suggests that there are alternatives, such as condom catheters which reduce associated UTIs and increase patient comfort. Novel technologies must be designed to block catheter associated urinary infections by either the extraluminal or intraluminal routes or both.

\section{Development of high-strength drug-eluting biodegradable stents by additive manufacturing}

\author{
Chaitra Venkatesh $^{1 *}$, Ian Major ${ }^{1}$, John G. Lyons ${ }^{2}$, \\ Declan M. Devine ${ }^{1}$ \\ ${ }^{1}$ Material Research Institute, Athlone Institute of Technology, \\ Ireland; ${ }^{2}$ Faculty of Engineering and Informatics, Athlone Institute \\ of Technology, Ireland
}

Biodegradable polymer nanocomposites are considered to be good alternatives in urology which provide support before they are absorbed by the body over a period of time. In this study, an innovative biodegradable nanocomposite is developed by using polylactic acid (PLA) as the base polymer, and is reinforced with non-toxic nanoclay, halloysite nanotubes (HNTs) by the extrusion process. These materials are selected as they are widely used in the biomedical field due to their biodegradability, natural availability and high mechanical properties.
Thus far, the effect of screw-speed during extrusion, drug-loading and drug-release kinetics, and 3D printing of the nanocomposite into model medical implants has been examined. The increase in screw speeds during extrusion resulted in significant increase in strength, toughness and thermal properties by better dispersion of the nanoclay in the polymer matrix as hydrogen-bonding interactions occur between the materials.

Further, for the drug release study, HNTs were first encapsulated with a model drug Aspirin. Then, the drug loaded HNTs of both ratios were blended into PLA matrix via melt-extrusion to form drug-loaded nanocomposite films. For comparison, the HNTs, PLA and Aspirin were tumble-mixed and processed as a separate batch. The results indicate the improved mechanical durability, drug-loading efficiency and sustained drug-release profile.

The nanocomposite was successfully extruded into filaments of $1.75 \pm 0.10 \mathrm{~mm}$ diameter in-house using twin screw extruder with a specialized die fixed to the extruder for the manufacture of production grade 3D printing filament. This resultant filament was utilized for Fused Filament Fabrication (FFF) into standard tensile test bars and $25 \mathrm{~mm}$ model medical implants using a standard FFF printing machine. The nanocomposite was successfully 3D printed into model medical implants.

\section{Influence of chronic kidney disease on in situ tissue formation in vascular access grafts}

Paul J. Besseling ${ }^{1}$, M. M. Krebber ${ }^{1}$, J. O. Fledderus ${ }^{1}$, M. Teraa ${ }^{1}$, A. Lichauco $^{3}$, A. Serrero ${ }^{3}$, C. V. C. Bouten ${ }^{2}$, P. Y. W. Dankers ${ }^{2}$, M. $\operatorname{Cox}^{3}$, M. C. Verhaar ${ }^{1}$

${ }^{1}$ Department of Nephrology and Hypertension, UMC Utrecht, Utrecht, The Netherlands; ${ }^{2}$ Department of Biomedical Engineering, and Institute for Complex Molecular Systems, TU/e, Eindhoven, The Netherlands; ${ }^{3}$ Xeltis BV, Eindhoven, The Netherlands

Vascular access is considered the Achilles' heel of haemodialysis, requiring frequent interventions to maintain patency. A proposed solution is self-healing vascular access grafts by means of in situ tissue engineering. One major limitation is the reliance upon the presence of a functional wound healing/immune response that has a sufficient capacity to initiate regeneration/healing. However, this is negatively affected by chronic kidney disease (CKD). Therefore, we aim to study the effect of CKD on in situ tissue formation in vascular access grafts in a rat model.

To mimic the effect of CKD in humans a rat model is created by 5/6th nephrectomy. Control animals receive a sham operation. When CKD animals reach a threshold of $50 \mathrm{mg} / 24 \mathrm{~h}$ proteinuria a vascular graft is implanted by end-to-end interposition in the abdominal aorta. Explantation is performed at 2, 4, 8 and 12 weeks. Explants are monitored for cell infiltration, proliferation, immune-, endothelial-, smooth muscle cells presence and ECM components.

Initial results indicate that after 2 weeks all implanted grafts contain numerous infiltrated cells, but no endothelial or smooth muscle cells. No difference is observed in the number of infiltrating or dividing cells between grafts from CKD and control animals. Results from later time points and immune analysis are expected in the coming months.

It is concluded that, after 2 weeks, CKD does not seem to alter early cell infiltration in the grafts. Later time points will be essential in the investigation of the wound healing process in CKD and its effects on tissue formation. 


\section{Development and experimental assessment of a novel biodegradable, anti-reflux and heparin- coated ureteral stent: the BraidStent}

\section{Julia E. de la Cruz ${ }^{1 *}$, Cristina Sousa ${ }^{1}$, Alberto Budia ${ }^{2}$, Luis Resel-Folkersma ${ }^{3}$, Francisco Sánchez-Margallo ${ }^{1}$, Federico Soria ${ }^{1}$}

${ }^{1}$ Jesús Usón Minimally Invasive Surgery Centre (JUMISC), Caceres, Spain; ${ }^{2}$ Urology Department, La Fe University Hospital, Valencia, Spain; ${ }^{3}$ San Carlos Clinical Hospital, Madrid, Spain

The objective of this study is the development and experimental assessment of a biodegradable, intra-ureteral, anti-reflux and heparincoated ureteral stent (BraidStent).

A sample size of 36 swine animal models was used. After baseline evaluation of the urinary tract, the animals were distributed into 3 homogeneous groups: Group-I, unilateral placement of the BraidStent (3Fr); Group-II, iatrogenic ureteroscopic perforation model and BraidStent stenting; Group-III, ureteral stricture model, treated via laser endoureterotomy and BraidStent disposal. Intermediate followups were carried out at 1,3 and 6 weeks and the final follow-up at 5 months. During baseline evaluation and follow-ups the internal diameter of the UPJ, ureteral peristalsis, urinoma, vesicoureteral reflux (VUR), migration, damage to the ureteral orifice, urothelial hyperplasia and obstructive effects of the stent fragments were assessed. The diagnostic tests performed for these assessments were: blood and urinalysis, ultrasonography, fluoroscopy, cystoscopy, ureteroscopy and simulated voiding cystourethrography.

Statistical significance was found in UPJ diameters between the baseline evaluation and the follow-ups, evidencing the occurrence of passive ureteral dilation. Treatment success rates were 95.8 and $83 \%$ in Groups II and III, respectively. Overall stent migration rate at 3 weeks was $16.6 \%$ and positive uroculture rate was $46 \%$. Ureteral peristalsis distal to the BraidStent was maintained in $80.3 \%$ of the ureters at 6 weeks. None of the animals showed VUR at any phase of the study. BraidStent degrades completely in all cases between 3 and 6 weeks, with no evidence of obstructive phenomena.

BraidStent biodegradable ureteral stent shows a predictable biodegradation rate, without obstructive fragments during its hydrolytic degradation process. The use of this stent results in passive ureteral dilation with no VUR. Therefore, it is likely that tolerance to this stent by patients will be better than to current stent designs.

\section{Polyurethane-based supramolecular hydrogels as delivery platforms for hydrophobic drugs}

\author{
Alessandro Torchio*, Monica Boffito, Gianluca Ciardelli
}

Politecnico di Torino, Department of Mechanical and Aerospace Engineering (DIMEAS), Turin, Italy

In this work, supramolecular (SM) hydrogels based on polyurethanes (PUs) and cyclodextrins (CDs) were synthesised for advanced drug delivery applications. These systems exhibit hierarchically selfassembled structures consisting of water-suspended channel-type crystals provided by poly(pseudo)rotaxanes. Indeed, in aqueous solutions PUs containing poly(ethylene oxide) (PEO) domains can interact with CDs, which can thread along PU chains and form poly(pseudo)rotaxanes. These structures can then assemble in the form of physical crystals and induce a sol-to-gel transition, thus forming supramolecular hydrogels. In these systems, free CDs can be exploited to encapsulate and release hydrophobic drugs with antiinflammatory or anti-bacterial properties.

Two PUs were synthesized from Poloxamer 407 (70\% wt PEO, $30 \%$ wt poly(propylene oxide) (PPO)) [Boffito et al., 2016, https://doi.org/10.1002/pi.5080]. SM gels were produced by mixing PU and CD aqueous solutions. The developed gels showed prolonged stability in contact with physiological-like aqueous environments (i.e., $\mathrm{pH} 7.4$ and at $37^{\circ} \mathrm{C}$ ) and turned out be thixotropic and selfhealing, thus demonstrating that CDs are a key factor in the design of hydrogels with a low synthetic polymer content. The hydrogels showed dynamic behaviour when incubated at $37{ }^{\circ} \mathrm{C}$ in the presence of aqueous solutions, releasing a great number of free CDs for several days without showing cytotoxicity (according to ISO10993).

The physical properties of the designed gels make them promising systems for an easy integration into biomedical devices, such as ureteral stents, aiming at localized and controlled drug release. For instance, these shape-adaptive hydrogels could be loaded in stent devices with specifically designed reservoirs, thus allowing the release of encapsulated drugs for the treatment of common clinical complications, such as infections and inflammations. Additionally, PU-based SM hydrogels showed enhanced mechanical properties and stability compared to P407-based SM systems already characterized in the literature [Simões et al. 2012, https://doi.org/10.1016/ j.ejpb.2011.09.017].

\section{Reference}

Simões SM, Veiga F, Torres-Labandeira JJ, Ribeiro AC, Sandez-Macho MI, Concheiro A, Alvarez-Lorenzo C (2012) Syringeable Pluronic- $\alpha$ cyclodextrin supramolecular gels for sustained delivery of vancomycin. Eur J Pharm Biopharm 80(1):103-112. https://doi.org/10.1016/ j.ejpb.2011.09.017

Publisher's Note Springer Nature remains neutral with regard to jurisdictional claims in published maps and institutional affiliations. 